\title{
Current and future approaches in the prevention and treatment of diabetic retinopathy
}

\author{
Louis K Chang' \\ David Sarraf',2 \\ 'Jules Stein Eye Institute, \\ Department of Ophthalmology, \\ University of California, Los Angeles, \\ Los Angeles, CA, USA; ${ }^{2}$ Department \\ of Ophthalmology, Greater LA VA \\ Healthcare Center, Los Angeles, CA
}

Correspondence: David Sarraf Jules Stein Eye Institute, UCLA, 100 Stein Plaza, Los Angeles, CA 90095, USA

Tel: + I 3108253090

Fax: + | $310825044 \mid$

Email dsarraf@ucla.edu

\begin{abstract}
Diabetic retinopathy (DR) is a major cause of blindness worldwide and is the number one cause of blindness in working-age individuals in developed countries. We review the current literature and discuss the pathogenesis, modifying risk factors, genetics, and treatment of DR. Special focus is placed on the rationale and effectiveness of therapeutic modalities, both current and future.
\end{abstract}

\section{Introduction}

Diabetes mellitus (DM) is a major cause of morbidity and mortality worldwide, especially in developed nations. Worldwide 135 million suffer from DM and in the United States alone, roughly 18 million individuals are affected with DM, 4.1 million of whom have sight-threatening diabetic retinopathy (DR). Complications from DR account for the leading cause of vision loss in the US working population (Klein et al 1984a; Klein et al 1984b).

Clinically, DR can be classified as nonproliferative or proliferative diabetic retinopathy (NPDR and PDR, respectively). Signs of NPDR include retinal microaneursyms, intraretinal dot and blot hemorrhages (DB), hard exudates (HE), and cotton wool spots (CWS). Intraretinal microvascular abnormalities (IRMA) and venous beading (VB) may be seen in severe NPDR or preproliferative diabetic retinopathy (Chew and Ferris 2006). Signs of PDR include retinal and optic disc neovascularization, which may cause preretinal, subhyaloid, and vitreous hemorrhage (VH) and tractional retinal detachment (TRD).

DR accounts for the vast majority of diabetes-related vision impairment. Microvascular damage increases retinal vascular permeability and may lead to diabetic macular edema (DME). The increased permeability allows egress of fluid and plasma components, leading to thickening of the macula and lipoprotein deposition. DME prevalence increases with the grade of retinopathy and is the most common cause of vision loss in NPDR (Chew and Ferris 2006). Capillary closure within the macula may lead to foveal avascular zone enlargement or macular ischemia. VH and/or RD may complicate PDR; the former is the most common cause of blindness in diabetics (Klein et al 1984a; Klein et al 1984b). With extensive ischemia, neovascularization may occur in the iris and occlude the angle leading to neovascular glaucoma, which has a high-risk of severe, irreversible vision loss.

Along with macular edema and ischemia, cell dysfunction and/or death may contribute to visual impairment in the setting of DM. This concept is supported by the finding that, in addition to greater severity of DR and increased fluorescein leakage, decreased summed electroretinographic oscillatory potentials was found to be an independent predictor of progression to severe PDR (Bresnick and Palta 1987a, 1987b). Moreover, increased apoptosis of cells in all layers of the retina has been observed in animal models of DR and in post-mortem analysis of retinas from diabetic 
patients (Barber et al 1998). However, it is unclear whether this increased cell death directly causes retinal dysfunction or represents loss of hypo- or non-functional cells.

\section{Pathogenesis}

Hyperglycemia leads to microvascular retinal disease through various postulated mechanisms, including the polyol pathway (Robinson et al 1983), advanced glycation end products (AGE) pathway (Brownlee et al 1988), the renin-angiotensinogen pathway (Deinum et al 1990), the angiogenesis pathway (especially that associated with vascular endothelial growth factor or VEGF) (Ferrara 1995), and pathways associated with oxidative damage (Kunisaki et al 1995). The first mechanism, the polyol pathway, is no longer considered an important target for the therapy of DR. Hyperglycemia leads to an increase in aldose reductase enzyme activity and increased sorbitol production causing lens and retinal toxicity by various mechanisms including osmotic effects, breakdown of the blood-retina barrier, and loss of pericytes ( Engerman and Kern 1984; Cheung et al 2005). Aldose reductase inhibitors have shown promise in animal models of DR, but human studies have had limited success (Anonymous 1993b). The other pathogenic pathways hold more promise for future therapies and these are discussed later in this review.

\section{Risk factors for the progression of diabetic retinopathy}

Risk factor modification is the single most important intervention in decreasing the rate of development and progression of DR. Although the duration of diabetes is a significant risk factor, hyperglycemia and hypertension are the most critical modifiable risk factors (Jain et al 2003). The Wisconsin Epidemiologic Study of Diabetic Retinopathy (WESDR) conducted a cross sectional population survey and found a linear relationship of $\mathrm{A} 1 \mathrm{C}$ level correlated with retinopathy ie,the lower the $\mathrm{A} 1 \mathrm{C}$ level the lower the prevalence of retinopathy (Klein et al 1988). The Diabetic Control and Complications Trial (DCCT) showed that type I diabetics prospectively randomized to intensive glycemic therapy to maintain $\mathrm{HgAlC}$ below $7 \%$ had a $76 \%$ reduction in development of diabetic retinopathy, $47 \%$ reduction in the incidence of severe NPDR or PDR, 23\% decreased incidence of macular edema, 54\% reduction in the rate of progression of pre-existing diabetic retinopathy, and a $56 \%$ decrease in the need for laser therapy (Anonymous 1993a). A long-term sustained benefit of initial intensive glycemic control was demonstrated by the Epidemiology of Diabetes Interventions and Complications Trial
(EDIC), an extension of the DCCT with study length of over 10 years (Anonymous 2000b).

The United Kingdom Prospective Diabetes Study (UKPDS) confirmed a benefit of intensive glycemic control in decreasing the risk of progression of diabetic retinopathy in older type II diabetics (Anonymous 1998). Subsequent analysis showed that among diabetics who also had hypertension, tighter blood pressure control $(<150 / 85)$ was as important as glycemic control. Diabetic retinopathy progression and visual acuity decline were reduced by $34 \%$ and $47 \%$ respectively versus those in the control group (blood pressure 180/105) (Anonymous 1998b).

A number of reports have identified elevated serum lipids as a risk factor for the progression of various features of DR. The WESDR and the ETDRS showed an association between elevated total serum lipid and cholesterol levels and increased hard exudates (Klein et al 1991; Chew et al 1996), a significant risk factor for moderate vision loss. Subsequent analysis showed that the presence of severe hard exudates was the strongest predictive factor in the development of subretinal fibrosis, an adverse clinical finding, in patients with DME (Fong et al 1997). Elevated serum triglycerides were also found to be a risk factor for development of high-risk PDR (Davis et al 1998). In a cohort of type 1 diabetics, a positive correlation between DR progression and elevated serum triglycerides and negative correlation with serum HDL levels was identified (Lyons et al 2004). Higher serum cholesterol and triglyceride levels increased the risk for progression of retinopathy while total and LDL cholesterol were positively associated with more severe retinal HE (van Leiden et al 2002). In a prospective, double-blind randomized control trial, reduction of total and LDL cholesterol with simvastatin was associated with a reduction in worsening of vision from in type 1 or 2 diabetics (Sen et al 2002).

Pregnancy has been linked to the more rapid progression of DR (Chew et al 1995). Either poorer or paradoxically tighter glycemic control during pregnancy may lead to more rapid progression of DR. Systemic changes (eg, hormonal and cardiovascular), may also account for this observation (Anonymous 2000a). Nevertheless, improved blood glucose control and more stringent DR screening criteria are recommended for pregnant diabetics.

Microalbuminuria and proteinuria indicative of diabetic nephropathy have been identified as risk factors for DR progression in some, but not all, studies. Importantly, this may not be a causal relationship, but rather two sequelae of generalized angiopathy caused by changes in enzymatic reduction of heparin sulfate proteoglycan side chains (Deckert et al 1989). Danaparoid sodium, a mixture of 
sulfated glycosaminoglycans with mainly heparan sulfate, improved proteinuria in type 1 diabetics (Gambaro 1998), but did not improve proteinuria or retinopathy among type 2 diabetics (van der Pijl et al 1999).

The incidence of cataract is higher among diabetics than the general population and cataract extraction may be required to improve visual function and/or to improve the view to the retina for diagnostic and therapeutic purposes. DR may limit the visual prognosis after cataract extraction, especially in the presence of severe NPDR or PDR (Chew et al 1999). While newer phacoemulsification techniques are associated with a lower incidence of DR progression, post-operative macular edema may still limit visual outcomes after cataract surgery (Krepler et al 2002). An association was found between progression of NPDR and rapid pre-operative glycemic control, suggesting that cataract surgery should be delayed until prolonged glycemic control has been achieved (Suto et al 2006).

In the US, African American and Latino populations have an increased incidence and severity of DR that cannot be explained by glycemic control alone. The great variability in severity of DR among populations with similar environmental factors has fueled the search for genetics factors that may contribute to the development of DR (Uhlmann et al 2006). Variations at several genetic loci have been implicated in DR, including VEGF, $\alpha 2 \beta 1$ integrin, ALR2, angiotensin II receptor 1, RAGE, and PPAR $\gamma 2$ (Uhlmann et al 2006). However, the interpretation of the results of many of these studies is confounded by conflicting reports that have failed to confirm such an association and the limited racial diversity of each of the study populations.

\section{Conventional treatments}

Traditional treatment of diabetic retinopathy has been shaped by the results of a few large prospective clinical trials. The Early Treatment Diabetic Retinopathy Study (ETDRS) sought to determine the efficacy of photocoagulation in the treatment of diabetic macular edema (Anonymous 1991). Patients with mild, moderate, or severe NPDR or lowrisk PDR were randomized to immediate or delayed focal macular laser (FML) and immediate or delayed panretinal laser photocoagulation (PRP) for the treatment of clinically significant macular edema (CSME). Immediate FML decreased moderate vision loss by $50 \%$ in patients with CSME, while immediate PRP with delayed FML in patients with NPDR and CSME responded least favorably. The study group concluded that FML for CSME should be performed before scatter PRP in patients with NPDR. In patients with high-risk PDR and CSME, eyes should receive immediate
PRP beginning nasally and inferiorly (to limit progression of macular edema) concurrently with FML.

The Diabetic Retinopathy Study (DRS) was designed to determine the efficacy of PRP in preventing severe visual loss in eyes with advanced DR (Anonymous 1981). Study subjects with bilateral PDR or severe NPDR (ie, preproliferative DR) were given PRP in the first eye and no treatment in the fellow eye. PRP reduced the risk of severe vision loss by $50 \%$ or more in preproliferative and proliferative retinopathy (7\% versus 3\%). The effect was most marked in high risk PDR (26\% versus 11\%), as defined by the presence of any neovascularization of the disc (NVD) or retina with $\mathrm{VH}$, or severe NVD with or without VH. The authors concluded that for severe NPDR, PDR, and especially high risk PDR, PRP is essential in preventing blindness. Side effects of treatment included peripheral visual field constriction and nyctalopia. Additional complications of PRP include exacerbation of DME and contraction of retinal fibrosis, exacerbating TRD and/or causing retinal breaks.

The Diabetic Retinopathy Vitrectomy Study (DRVS) was designed to compare early versus delayed pars plana vitrectomy (PPV) in patients with PDR, active neovascularization, and vitreous hemorrhage (Anonymous 1988). Patients with Type 1 DM, but not Type II DM, with dense non-clearing vitreous hemorrhage, had better outcomes with early vitrectomy. The study group recommended early PPV for vitreous hemorrhage in the setting of Type 1 DM or for eyes with useful vision and advanced active PDR. An important caveat to this study is that endolaser (intraoperative PRP) was not used in this study, possibly limiting the applicability of the results to current clinical practice.

Although practice patterns vary widely, current indications for vitrectomy in the setting of DR include non-clearing VH, TRD threatening or involving the macula, or combined tractional and rhegmatogenous retinal detachment. A number of techniques may be employed for TRD repair, including the use of long-acting intraocular gas or silicone oil, that may improve anatomic and functional outcomes (Mason et al 2006). Vitrectomy has also been used in the treatment of recalcitrant DME, especially when a tractional component is present, although its benefit has not been proven in a randomized, controlled clinical trial (see below).

\section{Treatments currently under clinical investigation}

Despite these proven treatments for retinal complications of diabetes, patients with diabetic retinopathy continue to experience visual loss. A number of off-label non-FDA 
approved treatment modalities are currently in clinical use and/or are under investigation for the treatment of various aspects of DR.

\section{Corticosteroids}

Periocular and intraocular triamcinolone acetonide (TA) have been used for the treatment of macular edema, including DME. This effect may be most pronounced in cases of cystoid macular edema (CME) or diffuse macular edema recalcitrant to laser therapy (Jonas et al 2001). A small randomized prospective clinical trial showed a beneficial effect of intravitreal TA (Jonas et al 2006). 40 eyes with diffuse DME were randomized to receive a single $20 \mathrm{mg}$ intravitreal TA or sham injection. At 3 and 6 months, $81 \%$ and $87 \%$ of the eyes in the TA group had greater than a 2 line improvement in visual acuity versus less than $30 \%$ in the control group. A benefit was also seen in a randomized, controlled prospective trial of intravitreal TA for refractory DME with two-year follow-up (Gillies et al 2006). 69 eyes were randomized to receive sham or intravitreal TA injections ( $4 \mathrm{mg}$ ), repeated up to five times. Participants in both groups were eligible to receive focal photocoagulation if deemed necessary. At the end of the study, TA-treated eyes had a decreased mean retinal thickness and improved visual outcome, with a $>5$ line improvement in $56 \%$ or eyes, versus $26 \%$ in the placebo group.

A prospective trial comparing intravitreal TA to macular grid photocoagulation for the treatment of CME showed that TA was more effective than focal laser in this setting (Avitabile et al 2005). 48 patients with DME were randomized to receive photocoagulation, $4 \mathrm{mg}$ TA, or both. The TA group had improved visual acuity and decreased retinal thickness when compared to the photocoagulation group. The group receiving both TA and photocoagulation was similar to TA alone. The Diabetic Retinopathy Clinical Research (DRCR) study group is currently evaluating TA versus photocoagulation for the treatment of DME.

Triamcinolone has also been used as an adjunctive treatment to FML or PRP, to potentiate the effect of focal or panretinal photocoagulation or to minimize the development or progression of macular edema (Kaderli et al 2005; Bandello et al 2006; Zein et al 2006; Choi et al 2007; Iida 2007; Lam et al 2007). Despite the clear benefits of steroid therapy these must be judged against significant side effects which include local problems such as redness, discomfort and floaters in the first week after the injection and a high incidence of increased intraocular pressure and cataract. Blinding complications such as endophthalmitis, vitreous hemorrhage, or retinal vascular occlusion are fortunately very rare (Jonas et al 2006).

To circumvent the need for repeated injections, a number of sustained release devices are under investigation. A dexamethasone drug delivery system (Posurdex DDS, Dexamethasone Posterior Segment Drug Delivery System, Allergan) consists of a biodegradable copolymer of lactic acid and glycolic acid which releases dexamethasone for up to 6 months into the vitreous cavity. In a randomized control trial of the DDS for the treatment of persistent macular edema involving 315 patients with macular edema, 152 of whom had DME, use of a 700 implant $\mu \mathrm{g}$ was associated with a higher rate of 10 and 15-letter improvement in visual acuity, decreased fluorescein leakage, and decreased retinal thickness (Kuppermann et al 2007). While there was an increased rate of IOP elevation in the treatment group, no eyes required glaucoma surgery or laser.

Other steroid delivery devices are being investigated for the treatment of ocular inflammation, but may eventually find use in the management of DME. Retisert (Control Delivery Systems and Bausch and Lomb) is an implantable nonbiodegradable intraocular device that delivers fluocinolone acetonide and is FDA approved for the treatment of noninfectious posterior uveitis (Jaffe et al 2006). Importantly, during the 34 -week trial, over $50 \%$ of patients required antihypertensive eyedrops and almost $6 \%$ required glaucoma filtering surgery and nearly $20 \%$ experienced significant cataract progression, $10 \%$ requiring cataract surgery. Thus, while such delivery devices may circumvent the need for repeated injections, they are still subject to significant side effects.

The exact mechanisms by which steroids affects vascular permeability are not known. Downregulation of inflammatory mediators and angiogenic cytokines has been demonstrated in various in vitro models (Antonetti et al 1998, 2002; Nauck et al 1998). However, a decrease in macular edema, as determined by OCT, was observed as soon as one hour after intravitreal injection of TA, arguing that nongenomic effects, such as direct restoration of the blood-retina barrier or modulation of membrane channels, contribute to its salutary effects (Felinski and Antonetti 2005; Miyamoto et al 2006).

\section{Vascular endothelial growth factor (VEGF)}

VEGF has a central role in vascular permeability and angiogenesis in neovascular age-related macular degeneration. VEGF has also been implicated in DR as an important angiogenic growth factor produced by areas of ischemic 
retina. Vitreous levels of VEGF progressively increase with more advanced retinopathy and are greatest in eyes with PDR and least in eyes from non-diabetic individuals or diabetics without DR (Adamis et al 1994; Aiello et al 1994). A reduction of vitreous VEGF levels has been documented with PRP (Aiello et al 1994). VEGF levels have also been correlated with degree of macular edema, as determined by degree of leakage with fluorescein angiography and central retinal thickness by OCT imaging (Funatsu et al 2006).

A prospective, double-masked clinical trial using pegaptanib sodium (Macugen, Eyetech), an aptamer that inhibits VEGF 165 isoform, for DME has been completed. (Cunningham et al 2005) One-hundred and seventy-two patients with DME and BCVA between 20/50 and 20/320 were randomized to receive $0.3 \mathrm{mg}, 1 \mathrm{mg}$, or $3 \mathrm{mg}$ intravitreal pegaptanib injections or sham injections. Treatments were given at study entry, six weeks, and twelve weeks followed by additional injections with or without FML as needed for another 18 weeks. At the end of the 36-week trial, subjects in the $0.3 \mathrm{mg}$ pegaptanib group had improved median BCVA (20/50 versus $20 / 63$ in the sham group), and more eyes gained $>10$ letters ( $24 \%$ vs. $10 \%)$ and $>15$ letters ( $18 \%$ vs. $7 \%$ ). Mean central retinal thickness as determined by OCT decreased by 68 um, while it decreased by only 4 um in the control group. Photocoagulation was deemed necessary in $25 \%$ of pegaptanib-treated eyes, while $48 \%$ of the control eyes received FML. No additional benefit was seen with the higher doses of pegaptanib. The pegaptanib treatment was well tolerated, with only one case of culture-negative endophthalmitis reported.

Further analysis examined the effects of VEGF-inhibition on PDR. Sixteen eyes in this study group had proliferative diabetic retinopathy at study entry. Of the 13 eyes in the pegaptanib treatment arm, eight (62\%) showed regression of neovascularization based on fundus photography or fluorescein angiography. Of the 4 fellow eyes and 3 eyes from the control group with baseline neovascularization that were examined, none showed regression. Further evidence for a direct effect on proliferative changes from diabetic retinopathy was the progression in 3 of the eight eyes which had regressed on pegaptanib therapy at week 52 after discontinuing pegaptanib at week 30 .

Ranibizumab (Lucentis, Genentech) is a neutralizing antibody targeted against all isoforms of VEGF which is FDA-approved for the intravitreal treatment of all subtypes of neovascular AMD. In a nonrandomized pilot study of ten patients, eyes with chronic DME received intraocular injections of $0.5 \mathrm{mg}$ ranibizumab at study entry and at
1, 2, 4, and 6 months (Chun et al 2006). At 7 months, mean visual acuity improved from $20 / 80$ to $20 / 40$ and mean foveal thickness decreased from $503 \mathrm{um}$ to $246 \mathrm{um}$. No adverse events, including increase in foveal capillary nonperfusion, were reported.

Bevacizumab (Avastin, Genentech) is a humanized panVEGF inhibiting antibody approved for colorectal cancer therapy. Whereas ranibizumab consists only of the Fc component of the antibody, bevacizumab is the full antibody and is not FDA-approved for intraocular use. A prospective examination of 51 eyes with DME that received one or two injections of bevacizumab with 6 to 12 week follow-up has been reported (Haritoglou et al 2006). Mean retinal thickness decreased from $541 \mathrm{um}$ to $417 \mathrm{um}$ at 6 weeks and to $377 \mathrm{um}$ at 12 weeks. However, a statistically-significant improvement in visual acuity was only seen at the 6 week time point. Further analysis showed that baseline visual acuity was the only predictive factor for an increase in visual acuity after 6 weeks of treatment. A more pronounced effect was reported in a retrospective analysis of 78 eyes with DME treated with 1 to 3 intravitreal injections of bevacizumab (1.25 $\mathrm{mg}$ or $2.5 \mathrm{mg}$ ) (Arevalo et al 2007). Bevacizumab-treated eyes showed a decrease in mean retinal thickness, stabilization of visual acuity in $41 \%$ of eyes, and a 2 line improvement in $55 \%$ of patients after 6 months of follow-up.

Regression of iris, retinal and optic disc neovascularization clinically and angiographically after intravitreal administration of bevacizumab has been shown in a large retrospective case series, although eventual reperfusion of the neovascular fronds was documented in several cases (Avery et al 2006). In some cases, the effects of VEGF-inhibition on PDR was seen as soon as 24 hours after treatment. Although not substantiated by randomized controlled trials, reduction in the caliber and fragility of neovascular lesions with anti-VEGF agents may decrease intraoperative hemorrhage during anterior and posterior segment surgery in the setting of PDR (Chen and Park 2006; Krzystolik et al 2006).

Other approaches to modulate intraocular VEGF activity are being investigated. Intraocular injection of a VEGFneutralizing anti-sense oligonucleotide has shown regression of ischemia-induced iris neovascularization in an animal model (Bhisitkul et al 2005). The VEGF trap (Regeneron, Bayer) is a soluble decoy VEGF receptor currently under investigation in the treatment of colorectal cancer (Konner and Dupont 2004). It consists of the binding domains of VEGF receptors 1 and 2, fused to the Fc fragment of IgG with a significantly higher binding affinity than monoclonal antibodies such as bevacizumab and ranibizumab for 
VEGF-A. An exploratory study of the use of the VEGF trap in the treatment of five patients with DME showed a decrease in retinal thickness and improvement in visual acuity, without serious adverse events (Do et al 2007).

\section{Protein kinase $\mathrm{C}$}

Hyperglycemia is an important factor for the development and progression of diabetic retinopathy (Anonymous 1993, 1998a). Hyperglycemia activates protein kinase C (PKC), a family of approximately 13 enzymes, through VEGF by inducing synthesis of diacylglycerol (Aiello 2002). In animal studies, upregulation of the beta-isoform increases retinal vascular permeability and neovascularization (Aiello et al 1997; Xu et al 2004), while inhibition or genetic knockout reduces diabetes-induced retinal permeability and ischemiainduced neovascularization (Danis et al 1998; Suzuma et al 2002).

Ruboxistaurin mesylate (LY333531) is an oral PKC B-isozyme selective inhibitor that has been shown to ameliorate diabetic retinopathy in animal models and improve alterations in retinal blood flow from early diabetic retinopathy in human subjects. The Protein Kinase C Inhibitor Diabetic Retinopathy Study (PKC-DRS) was undertaken to determine whether ruboxistaurin reduces that rate of progression of nonproliferative diabetic retinopathy to proliferative retinopathy. (Aiello et al 2006) The results of the study did not support the primary hypothesis. However, when compared to the placebo group, the treatment group had a lower rate of moderate visual loss (5.5\% vs. 9\%), a greater likelihood to gain $>15$ letters from baseline and a reduction in the use of focal/grid photocoagulation by $26 \%$.

Such findings suggested that PKC B inhibition may decrease vascular permeability and DME. The Protein Kinase C Inhibitor Diabetic Macular Edema Study (PKC-DMES) was designed to determine the effect of orally-administered ruboxistaurin on DME (Anonymous 2007). In this phase III trial, 686 with DME that did not meet ETDRS-criteria for CSME were randomized to placebo or $4 \mathrm{mg}, 16 \mathrm{mg}$, or $32 \mathrm{mg} /$ day of ruboxistaurin. After 30 months, there was no statistically significant difference in the progression to sight-threatening DME or the need for focal/grid photocoagulation.

\section{Octreotide}

Several lines of evidence support a role for growth hormone in the progression of diabetic retinopathy (Wilkinson-Berka et al 2006). Two phase III studies of the insulin-like growth factor antagonist octreotide have been completed. In one study, monthly intramuscular injections of Sandostatin LAR reduced the progression of $\mathrm{DR}$, but failed to benefit visual acuity or macular edema. However, a second study of similar design failed to show a beneficial effect on any of the outcome measures examined.

\section{Vitrectomy}

In addition to its role in the treatment of proliferative DR and vitreous hemorrhage, vitrectomy has been used for the treatment of DME (Lewis et al 1992). Spontaneous resolution of DME accompanying PVD has been reported. In one study, PVD was more prevalent in diabetics without DME than with DME (Nasrallah et al 1988; Nasrallah et al 1989). Postulated mechanisms of action include relief of traction, removal of cytokines in the vitreous that promote vascular permeability and proliferation, and increasing oxygen diffusion to the retina. A retrospective analysis of 65 eyes undergoing PPV with membrane peeling for DME showed a progressive decrease in mean retinal thickness and an improvement in visual acuity over the four month follow-up period (Yamamoto et al 2001).

Highly-purified hyaluronidase (Vitrase, ISTA) has been studied in phase III clinical trials in the treatment of vitreous hemorrhage (Kuppermann et al 2005). While it reduced vitreous hemorrhage and allowed photocoagulation, it did not show an improvement in visual acuity when compared to control. Intraocular Vitrase has been used to induce posterior vitreous detachment, but its role in the treatment of DME is yet to be elucidated.

\section{Additional potential targets for future therapy c-Raf kinase}

The Ras/Raf/MAPK signaling pathway has been implicated in angiogenesis in many types of systemic tumors and associated with ocular neovascularization. VEGF has been identified as a downstream effector of this pathway. Intravitreal injection of an anti-raf-1-kinase antisense oligonucleotide inhibited neovascularization in a porcine animal model (Danis et al 2003). 17-aminoallylgeldanamycin, an inhibitor of this pathway currently under investigation as a systemic chemotherapeutic, has been shown to inhibit retinal neovascularization in a mouse model of hypoxia-induced proliferative retinopathy. (Kociok et al 2007)

\section{Erythropoietin}

Erythropoeitin is another hypoxia-induced angiogenic factor that has been implicated in the pathogenesis of DR. Studies 
have reported that vitreous levels of erythropoietin in eyes with PDR are significantly higher than control (Katsura et al 2005; Watanabe et al 2005). Aqueous levels of erythropoietin from eyes with DME were significantly higher than those with neovascular AMD or control eyes.(Jonas and Neumaier 2007) Another study found a stronger correlation between grade of PDR and erythropoietin than that with VEGF (Watanabe et al 2005).

\section{Carbonic anyhdrase}

Carbonic anhydrase I was recently identified as one of 117 proteins upregulated in vitreous samples of eyes with proliferative diabetic retinopathy using an unbiased, mass spectroscopy-based proteomic analysis (Gao et al 2007). In an animal model, carbonic anhydrase I increased retinal vascular leakage and retinal thickness through a kallikrein and bradykinin-dependent pathway. Acetazolamide, a carbonic anhydrase inhibitor, is widely used for treatment of glaucoma and cystoid macular edema in the setting of retinitis pigmentosa. Previously, in a small pilot case-control study of 12 diabetics, oral acetazolamide improved angiographic edema, but had an equivocal benefit for visual acuity (Giusti et al 2001). Further study is needed to validate the biological and clinical importance of this pathway in DR and DME.

\section{Benfotiamine}

Benfotiamine, a thiamine derivative, inhibits three biochemical pathways of hyperglycemia-induced endothelial dysfunction, namely the hexosamine, AGE formation, and diacylglycerol-PKC pathways (Brownlee 2001). When administered to streptozocin-treated diabetic rats, benfotiamine decreased capillary changes from diabetic retinopathy (Hammes et al 2003). This treatment modality is currently under investigation for treatment of diabetic peripheral neuropathy (Haupt et al 2005) and holds promise in the prevention of DR.

\section{Conclusions}

DR remains a challenging clinical entity, both in terms of prevalence and morbidity. Laser photocoagulation remains the gold standard of care for vision-threatening complications of DR. Newer treatment modalities, especially triamcinolone and anti-VEGF therapy, are currently in widespread use, but there are no consensus guidelines regarding their use. Further understanding of the environmental, genetic, and biochemical factors that contribute to the development and progression of DR will continue to yield promising new targets for more effective and better-tolerated treatments. Despite current and future advances, prevention of DR by optimizing control of modifiable risk factors, especially glycemic and blood pressure control, will undoubtedly continue to have the most important role in the control of this disease process.

\section{Methods}

A comprehensive literature search was performed using the PubMed database. Initial search terms included "diabetes," "diabetic retinopathy," and "macular edema."

\section{Support}

Supported in part by RPB grant \#OP31 (David Sarraf).

\section{References}

Adamis AP, Miller JW, Bernal MT, et al. 1994. Increased vascular endothelial growth factor levels in the vitreous of eyes with proliferative diabetic retinopathy. Am J Ophthalmol, 118:445-50.

Aiello LP, Avery RL. Arrigg PG, et al. 1994. Vascular endothelial growth factor in ocular fluid of patients with diabetic retinopathy and other retinal disorders. $N$ Engl J Med, 331:1480-7.

Aiello LP, Bursell SE, Clermont A, et al. 1997. Vascular endothelial growth factor-induced retinal permeability is mediated by protein kinase $\mathrm{C}$ in vivo and suppressed by an orally effective beta-isoform-selective inhibitor. Diabetes, 46:1473-80.

Aiello LP. 2002. The potential role of PKC beta in diabetic retinopathy and macular edema. Surv Ophthalmol, 47(Suppl 2):S263-269.

Aiello LP, Davis MD, Girach A, et al. 2006. Effect of ruboxistaurin on visual loss in patients with diabetic retinopathy. Ophthalmology, 113:2221-30.

Anonymous. 1981. Photocoagulation treatment of proliferative diabetic retinopathy. Clinical application of Diabetic Retinopathy Study (DRS) findings, DRS Report Number 8. The Diabetic Retinopathy Study Research Group. Ophthalmology, 88:583-600.

Anonymous. 1991. Early photocoagulation for diabetic retinopathy. ETDRS report number 9. Early Treatment Diabetic Retinopathy Study Research Group. Ophthalmology, 98:766-85.

Anonymous. 1993a. The effect of intensive treatment of diabetes on the development and progression of long-term complications in insulindependent diabetes mellitus. The Diabetes Control and Complications Trial Research Group. N Engl J Med, 329:977-86.

Anonymous. 1993b. The sorbinil retinopathy trial: neuropathy results Sorbinil Retinopathy Trial Research Group. Neurology, 43:1141-9.

Anonymous. 1988a. Early vitrectomy for severe proliferative diabetic retinopathy in eyes with useful vision. Results of a randomized trial-Diabetic Retinopathy Vitrectomy Study Report 3. The Diabetic Retinopathy Vitrectomy Study Research Group. Ophthalmology, 95:1307-20

Anonymous. 1998b. Intensive blood-glucose control with sulphonylureas or insulin compared with conventional treatment and risk of complications in patients with type 2 diabetes (UKPDS 33). UK Prospective Diabetes Study (UKPDS) Group. Lancet, 352:837-53.

Anonymous. 2000a. Effect of pregnancy on microvascular complications in the diabetes control and complications trial. The Diabetes Control and Complications Trial Research Group. Diabetes Care, 23:1084-91.

Anonymous. 2000b. Retinopathy and nephropathy in patients with type 1 diabetes four years after a trial of intensive therapy. Am J Ophthalmol, 129:704-5.

Anonymous. 2007. Effect of ruboxistaurin in patients with diabetic macular edema: thirty-month results of the randomized PKC-DMES clinical trial. Arch Ophthalmol, 125:318-24. 
Antonetti DA, Barber AJ, Khin S, et al. 1998. Vascular permeability in experimental diabetes is associated with reduced endothelial occludin content: vascular endothelial growth factor decreases occludin in retinal endothelial cells. Penn State Retina Research Group. Diabetes, 47:1953-9.

Antonetti DA, Wolpert EB, DeMaio L, et al. 2002 Hydrocortisone decreases retinal endothelial cell water and solute flux coincident with increased content and decreased phosphorylation of occludin. $J$ Neurochem, 80:667-77.

Arevalo JF, Fromow-Guerra J, Quiroz-Mercado H, et al. 2007. Primary intravitreal bevacizumab (Avastin) for diabetic macular edema: results from the Pan-American Collaborative Retina Study Group at 6-month follow-up. Ophthalmology, 114:743-50.

Avery RL, Pearlman J, Pieramici DJ, et al. 2006. Intravitreal bevacizumab (Avastin) in the treatment of proliferative diabetic retinopathy. Ophthalmology, 113:1695 e1-15.

Avitabile T, Longo A, Reibaldi A. 2005. Intravitreal triamcinolone compared with macular laser grid photocoagulation for the treatment of cystoid macular edema. Am J Ophthalmol, 140:695-702.

Bandello F, Polito A, Pognuz DR, et al. 2006. Triamcinolone as adjunctive treatment to laser panretinal photocoagulation for proliferative diabetic retinopathy. Arch Ophthalmol, 124:643-50.

Barber AJ, Lieth E, Khin SA, et al. 1998. Neural apoptosis in the retina during experimental and human diabetes. Early onset and effect of insulin. J Clin Invest, 102:783-91.

Bhisitkul RB, Robinson GS, Moulton RS, et al. 2005. An antisense oligodeoxynucleotide against vascular endothelial growth factor in a nonhuman primate model of iris neovascularization. Arch Ophthalmol, 123:214-19.

Bresnick GH, Palta M. 1987a. Oscillatory potential amplitudes. Relation to severity of diabetic retinopathy. Arch Ophthalmol, 105:929-33.

Bresnick GH, Palta M. 1987b. Predicting progression to severe proliferative diabetic retinopathy. Arch Ophthalmol, 105:810-14.

Brownlee M. 2001. Biochemistry and molecular cell biology of diabetic complications. Nature, 414:813-20.

Brownlee M, Cerami A, Vlassara H. 1988. Advanced glycosylation end products in tissue and the biochemical basis of diabetic complications. N Engl J Med, 318:1315-21.

Chen E, Park CH. 2006. Use of intravitreal bevacizumab as a preoperative adjunct for tractional retinal detachment repair in severe proliferative diabetic retinopathy. Retina, 26:699-700.

Cheung, AK, Fung MK, Lo AC, et al. 2005. Aldose reductase deficiency prevents diabetes-induced blood-retinal barrier breakdown, apoptosis, and glial reactivation in the retina of $\mathrm{db} / \mathrm{db}$ mice. Diabetes, $54: 3119-25$

Chew EY, Mills JL, Metzger BE, et al. 1995. Metabolic control and progression of retinopathy. The Diabetes in Early Pregnancy Study. National Institute of Child Health and Human Development Diabetes in Early Pregnancy Study. Diabetes Care, 18:631-7.

Chew EY, Klein ML, Ferris FL $3^{\text {rd }}$, et al. 1996. Association of elevated serum lipid levels with retinal hard exudate in diabetic retinopathy. Early Treatment Diabetic Retinopathy Study (ETDRS) Report 22. Arch Ophthalmol, 114:1079-84.

Chew EY, Benson WE, Remaley NA, et al. 1999. Results after lens extraction in patients with diabetic retinopathy: early treatment diabetic retinopathy study report number 25. Arch Ophthalmol, 117:1600-6.

Chew EY, Ferris FLI. 2006. Nonproliferative diabetic retinopathy. In: Ryan, SJ (ed). Retina. Vol. II, Elsevier Mosby, St. Louis, pp. 1271-84.

Choi KS, Chung JK, Lim SH. 2007. Laser photocoagulation combined with intravitreal triamcinolone acetonide injection in proliferative diabetic retinopathy with macular edema. Korean J Ophthalmol, 21:11-17.

Chun DW, Heier JS, Topping TM, et al. 2006. A pilot study of multiple intravitreal injections of ranibizumab in patients with center-involving clinically significant diabetic macular edema. Ophthalmology, 113:1706-12.
Cunningham ET Jr., Adamis AP, Altaweel M, et al. 2005. A phase II randomized double-masked trial of pegaptanib, an anti-vascular endothelial growth factor aptamer, for diabetic macular edema. Ophthalmology, 112:1747-57.

Danis RP, Bingaman DP, Jirousek M, et al. 1998. Inhibition of intraocular neovascularization caused by retinal ischemia in pigs by PKCbeta inhibition with LY333531. Invest Ophthalmol Vis Sci, 39:171-9.

Danis RP, Criswell M, Orge F, et al. 2003. Intravitreous anti-raf-1 kinase antisense oligonucleotide as an angioinhibitory agent in porcine preretinal neovascularization. Curr Eye Res, 26:45-54.

Davis MD, Fisher MR, Gangnon RE, et al. 1998. Risk factors for high-risk proliferative diabetic retinopathy and severe visual loss: Early Treatment Diabetic Retinopathy Study Report \#18. Invest Ophthalmol Vis Sci, 39:233-52.

Deckert T, Feldt-Rasmussen B, Borch-Johnsen K, et al. 1989. Albuminuria reflects widespread vascular damage. The Steno hypothesis. Diabetologia, 32:219-26.

Deinum J, Derkx FH, Danser AH, et al. 1990. Identification and quantification of renin and prorenin in the bovine eye. Endocrinology, 126:1673-82.

Do DV, Nguyen QD, Browning DJ, et al. 2007. Results of a Phase I Study of Intravitreal VEGF Trap in Subjects With Diabetic Macular Edema: The CLEAR-IT DME Study. In: Association for Research in Vision and Ophthalmology, Fort Lauderdale, Florida.

Engerman RL, Kern TS. 1984. Experimental galactosemia produces diabetic-like retinopathy. Diabetes, 33:97-100.

Felinski EA, Antonetti DA. 2005. Glucocorticoid regulation of endothelial cell tight junction gene expression: novel treatments for diabetic retinopathy. Curr Eye Res, 30:949-57.

Ferrara N. 1995. Vascular endothelial growth factor. The trigger for neovascularization in the eye. Lab Invest, 72:615-18.

Fong DS, Segal PP, Myers F, et al. 1997. Subretinal fibrosis in diabetic macular edema. ETDRS report 23. Early Treatment Diabetic Retinopathy Study Research Group. Arch Ophthalmol, 115:873-7.

Funatsu H, Yamashita H, Nakamura S, et al. 2006. Vitreous levels of pigment epithelium-derived factor and vascular endothelial growth factor are related to diabetic macular edema. Ophthalmology, 113:294-301.

Gambaro G. 1998. Danaparoid sodium in diabetic retinopathy. Lancet, 351:674.

Gao BB, Clermont A, Rook S, et al. 2007. Extracellular carbonic anhydrase mediates hemorrhagic retinal and cerebral vascular permeability through prekallikrein activation. Nat Med, 13:181-88.

Giusti C, Forte R, Vingolo EM, et al. 2001. Is acetazolamide effective in the treatment of diabetic macular edema? A pilot study. Int Ophthalmol, 24:79-88.

Hammes HP, Du X, Edelstein D, et al. 2003. Benfotiamine blocks three major pathways of hyperglycemic damage and prevents experimental diabetic retinopathy. Nat Med, 9:294-9.

Haritoglou C, Kook D, Neubauer A, et al. 2006. Intravitreal bevacizumab (Avastin) therapy for persistent diffuse diabetic macular edema. Retina, 26:999-1005.

Haupt E, Ledermann H, Kopcke W. 2005. Benfotiamine in the treatment of diabetic polyneuropathy--a three-week randomized, controlled pilot study (BEDIP study). Int J Clin Pharmacol Ther, 43:71-7.

Iida T. 2007. Combined triamcinolone acetonide injection and grid laser photocoagulation: a promising treatment for diffuse diabetic macular oedema? Br J Ophthalmol, 91:407-8.

Jaffe GJ, Martin D, Callanan D, et al. 2006. Fluocinolone acetonide implant (Retisert) for noninfectious posterior uveitis: thirty-four-week results of a multicenter randomized clinical study. Ophthalmology, 113:1020-7.

Jain A, Sarraf D, Fong D. 2003. Preventing diabetic retinopathy through control of systemic factors. Curr Opin Ophthalmol, 14:389-94.

Jonas JB, Hayler JK, Sofker A, et al. 2001. Intravitreal injection of crystalline cortisone as adjunctive treatment of proliferative diabetic retinopathy. Am J Ophthalmol, 131:468-71. 
Jonas JB, Kamppeter BA, Harder B, et al. 2006. Intravitreal triamcinolone acetonide for diabetic macular edema: a prospective, randomized study. J Ocul Pharmacol Ther, 22:200-7.

Jonas JB, Kreissig I, Spandau UH, et al. 2006. Infectious and noninfectious endophthalmitis after intravitreal high-dosage triamcinolone acetonide. Am J Ophthalmol, 141:579-80.

Jonas JB, Neumaier M. 2007. Erythropoietin levels in aqueous humour in eyes with exudative age-related macular degeneration and diabetic retinopathy. Clin Experiment Ophthalmol, 35:186-7.

Kaderli B, Avci R, Gelisken O, et al. 2005. Intravitreal triamcinolone as an adjunct in the treatment of concomitant proliferative diabetic retinopathy and diffuse diabetic macular oedema. Combined IVTA and laser treatment for PDR with CSMO. Int Ophthalmol, 26:207-14.

Katsura Y, Okano T, Matsuno K, et al. 2005. Erythropoietin is highly elevated in vitreous fluid of patients with proliferative diabetic retinopathy. Diabetes Care, 28:2252-4.

Klein R, Klein BE, Moss SE, et al. 1984a. The Wisconsin epidemiologic study of diabetic retinopathy. III. Prevalence and risk of diabetic retinopathy when age at diagnosis is 30 or more years. Arch Ophthalmol, 102:527-32.

Klein R, Klein BE, Moss SE, et al. 1984b. The Wisconsin epidemiologic study of diabetic retinopathy. II. Prevalence and risk of diabetic retinopathy when age at diagnosis is less than 30 years. Arch Ophthalmol, 102:520-6.

Klein R, Klein BE, Moss SE, et al. 1988. Glycosylated hemoglobin predicts the incidence and progression of diabetic retinopathy. JAMA, 260:2864-71.

Klein BE, Moss SE, Klein R, et al. 1991. The Wisconsin Epidemiologic Study of Diabetic Retinopathy. XIII. Relationship of serum cholesterol to retinopathy and hard exudate. Ophthalmology, 98:1261-5.

Kociok N, Krohne TU, Poulaki V, et al. 2007. Geldanamycin treatment reduces neovascularization in a mouse model of retinopathy of prematurity. Graefes Arch Clin Exp Ophthalmol, 245:258-66.

Konner J, Dupont J. 2004. Use of soluble recombinant decoy receptor vascular endothelial growth factor trap (VEGF Trap) to inhibit vascular endothelial growth factor activity. Clin Colorectal Cancer, 4(Suppl 2):S81-5.

Krepler K, Biowski R, Schrey S, et al. 2002. Cataract surgery in patients with diabetic retinopathy: visual outcome, progression of diabetic retinopathy, and incidence of diabetic macular oedema. Graefes Arch Clin Exp Ophthalmol, 240:735-738.

Krzystolik MG, Filippopoulos T, Ducharme JF, et al. 2006. Pegaptanib as an adjunctive treatment for complicated neovascular diabetic retinopathy. Arch Ophthalmol, 124:920-1.

Kunisaki M, Bursell SE, Clermont AC, et al. 1995. Vitamin E prevents diabetes-induced abnormal retinal blood flow via the diacylglycerolprotein kinase C pathway. Am J Physiol, 269:E239-46.

Kuppermann BD, Thomas EL, de Smet MD, et al. 2005. Pooled efficacy results from two multinational randomized controlled clinical trials of a single intravitreous injection of highly purified ovine hyaluronidase (Vitrase) for the management of vitreous hemorrhage. Am J Ophthalmol, 140:573-84.

Kuppermann BD, Blumenkranz MS, Haller JA, et al. 2007. Randomized controlled study of an intravitreous dexamethasone drug delivery system in patients with persistent macular edema. Arch Ophthalmol, 125:309-17.

Lam DS, Chan CK, Mohamed S, et al. 2007. Intravitreal triamcinolone plus sequential grid laser versus triamcinolone or laser alone for treating diabetic macular edema six-month outcomes. Ophthalmology, Apr 23; [Epub ahead of print]
Lewis H, Abrams GW, Blumenkranz MS, Campo RV. 1992. Vitrectomy for diabetic macular traction and edema associated with posterior hyaloidal traction. Ophthalmology, 99:753-9.

Lyons TJ, Jenkins AJ, Zheng D, et al. 2004. Diabetic retinopathy and serum lipoprotein subclasses in the DCCT/EDIC cohort. Invest Ophthalmol Vis Sci, 45:910-18.

Mason JO 3rd, Colagross CT, Vail R. 2006. Diabetic vitrectomy: risks, prognosis, future trends. Curr Opin Ophthalmol, 17:281-5.

Miyamoto N, Iossifov D, Metge F, et al. 2006. Early effects of intravitreal triamcinolone on macular edema: mechanistic implication. Ophthalmology, 113:2048-53.

Nasrallah FP, Jalkh AE, Van Coppenolle F, et al. 1988. The role of the vitreous in diabetic macular edema. Ophthalmology, 95:1335-9.

Nasrallah FP, Van de Velde F, Jalkh AE, et al. 1989. Importance of the vitreous in young diabetics with macular edema. Ophthalmology, 96:1511-16.

Nauck M, Karakiulakis G, Perruchoud AP, et al. 1998. Corticosteroids inhibit the expression of the vascular endothelial growth factor gene in human vascular smooth muscle cells. Eur J Pharmacol, 341:309-15.

Robinson WGJ, Kador PF, Kinoshita JH. 1983. Retinal capillaries: basement membrance thickening by galactosemia preventede with aldose reductase inhibitor. Science, 221:1177-9.

Sen K, Misra A, Kumar A, et al. 2002. Simvastatin retards progression of retinopathy in diabetic patients with hypercholesterolemia. Diabetes Res Clin Pract, 56:1-11.

Suto C, Hori S, Kato S, et al. 2006. Effect of perioperative glycemic control in progression of diabetic retinopathy and maculopathy. Arch Ophthalmol, 124:38-45.

Suzuma K, Takahara N, Suzuma I, et al. 2002. Characterization of protein kinase C beta isoform's action on retinoblastoma protein phosphorylation, vascular endothelial growth factor-induced endothelial cell proliferation, and retinal neovascularization. Proc Natl Acad Sci U S A, 99:721-6.

Uhlmann K, Kovacs P, Boettcher Y, et al. 2006. Genetics of diabetic retinopathy. Exp Clin Endocrinol Diabetes, 114:275-94.

van der Pijl JW, Lemkes HH, Frolich M, et al. 1999. Effect of danaparoid sodium on proteinuria, von Willebrand factor, and hard exudates in patients with diabetes mellitus type 2. J Am Soc Nephrol, 10:1331-6.

van Leiden HA, Dekker JM, Moll AC, et al. 2002. Blood pressure, lipids, and obesity are associated with retinopathy: the hoorn study. Diabetes Care, 25:1320-5.

Watanabe D, Suzuma K, Matsui S, et al. 2005. Erythropoietin as a retinal angiogenic factor in proliferative diabetic retinopathy. $N$ Engl $J$ Med, 353:782-92.

Wilkinson-Berka JL, Wraight C, Werther G. 2006. The role of growth hormone, insulin-like growth factor and somatostatin in diabetic retinopathy. Curr Med Chem, 13:3307-17.

Xu X, Zhu Q, Xia X, et al. 2004. Blood-retinal barrier breakdown induced by activation of protein kinase $\mathrm{C}$ via vascular endothelial growth factor in streptozotocin-induced diabetic rats. Curr Eye Res, 28:251-6.

Yamamoto T, Akabane N, Takeuchi S. 2001. Vitrectomy for diabetic macular edema: the role of posterior vitreous detachment and epimacular membrane. Am J Ophthalmol, 132:369-77.

Zein, WM, Noureddin BN, Jurdi FA, et al. 2006. Panretinal photocoagulation and intravitreal triamcinolone acetonide for the management of proliferative diabetic retinopathy with macular edema. Retina, 26:137-42. 
